\title{
Fiber Dependence and Coupling Efficiency Limitation for a Lensed Fiber Integrated with a Long Period Fiber Grating
}

\author{
L. A. Wang and W. T. Chen \\ Institute of Electro-Optical Engineering, $R m .539$ \\ National Taiwan University, Taipei, Taiwan, R.O.C. \\ Email:lon@ccms.ntu,edu.tw \\ TEL:(886-2)23635251 ext 539 \\ FAX:(886-2)23656327
}

\begin{abstract}
We analyze the fiber dependence and the efficiency limitation for optical coupling using a lensed fiber integrated with a long period fiber grating. (C2000 Optical Society of America

OCIS codes: (060.2340) Fiber optics components; (230.0230) Optical devices
\end{abstract}

Long-period fiber gratings (LPFG's) have been demonstrated for various applications as band-rejection filters, gain equalizers, rocking filters, mode converters and sensors. Recently, we propose a new application by utilizing an LPFG with an integrated hemispherical lensed fiber to assist optical coupling [1-3]. Coupling efficiencies of $\sim 78 \%$ and $35 \%$ and the corresponding working distances of $\sim 250 \mu \mathrm{m}$ and $110 \mu \mathrm{m}$ are achieved when the lenses' radii of curvature of $\sim 50 \mu \mathrm{m}$ and $120 \mu \mathrm{m}$ and the $\mathrm{HE}_{11}$ cladding mode of the LPFG are used for the cases of fiber-to-fiber and laser diode (LD)-to-fiber, respectively. The main advantage of proposed coupling scheme is the resultant long working distance since the launched beam is coupled to the relatively large cladding area as opposed to the core area. A working distance longer than $\sim 100 \mu \mathrm{m}$ is generally desired for the packaging process of an optical sub-assembly module and for the reduction of optical back-reflection.

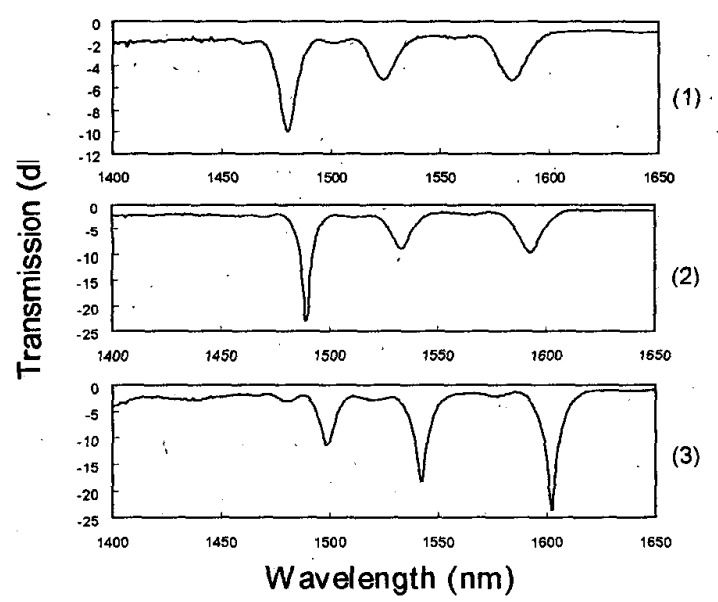

(a)

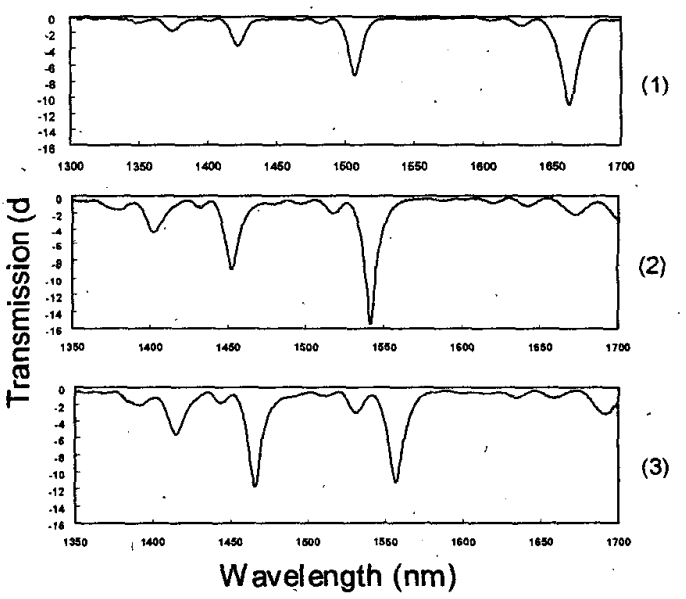

(b)

Fig. 1 Measured transmission spectra at exposure duration of (a)-(1) 2 mins, --(2) 3 mins, --(3) 3.5 mins for a DSF, and (b)-(1) 4 mins, -(2) 12 mins, -(3) 20 mins for a step-index SMF.

The fiber used in the proposed coupling scheme is a dispersion-shifted fiber (DSF) instead of a standard singlemode fiber (SMF). The transmission loss of $\mathrm{HE}_{11}$ mode is known to be very small, $\sim 1-3 \mathrm{~dB}$, for an LPFG imprinted in a step-index SMF owing to its weak coupling coefficient [4]. However; more than 25-dB transmission loss can be obtained when an LPFG is imprinted in a DSF. Therefore, we will compare the difference of electrical fields of the cladding modes of LPFG's for these two types of fibers and discuss their effects on the coupling efficiency. Figure 1(a) shows the measured transmission spectra of LPFG's in DSF's at three different exposure duration for 248-nm wavelength. The period and length of LPFG's are $450 \mu \mathrm{m}$ and $20 \mathrm{~mm}$, respectively. It is noted that the transmission loss of $\mathrm{HE}_{11}$ mode is higher than $\mathrm{HE}_{13}$ and $\mathrm{HE}_{15}$ modes at the exposure duration of $\sim 2$ minutes. As the duration increases, the transmission of $\mathrm{HE}_{11}$ mode increases rapidly and reaches a maximum of $\sim 20 \mathrm{~dB}$ while the transmission losses are only $-8 \mathrm{~dB}$ and $\sim 9 \mathrm{~dB}$ for $\mathrm{HE}_{13}$ and $\mathrm{HE}_{15}$ modes, respectively. The results are quite 
different as compared to the Figure 1(b), in which an LPFG is imprinted in a step-index fiber (Fibercore Corp.). A simulation is carried out to calculate the cladding modes of a DSF and a step-index SMF based on the finite difference method, in which a triangular-index profile is assumed for the DSF. Figure 2(a) and Figure 2(b) depict the normalized electric fields of $\mathrm{HE}_{11}, \mathrm{HE}_{13}$ and $\mathrm{HE}_{15}$ modes for a DSF and a step-index $\mathrm{SMF}$, respectively. It is noted that the electric field in the core is larger for $\mathrm{HE}_{11}$ than those for $\mathrm{HE}_{13}$ and $\mathrm{HE}_{15}$ modes for a DSF. Therefore, the core-to-cladding coupling coefficient is expected larger for $\mathrm{HE}_{11}$ [4]. Conversely, as - shown in Figure 2(b). the electric field in the core decreases as the order of cladding mode increases for a step-index SMF. It is also noted that the measured transmission of $\mathrm{HE}_{11}$ mode increases slowly and seems to saturate at a maximum of $\sim 2 \mathrm{~dB}$ for a long exposure duration of $\sim 20$ minutes, implying that a much smaller coupling efficiency than that can be obtained by using a DSF.

To obtain the coupling efficiency, we adopt the methodology that follows Reference 5, in which ray optics is assumed and the effects of fiber truncation, mode-mismatch, spherical aberration, and Fresnel reflection are considered. The total coupling efficiency, defined as $\eta_{\mathrm{m} \text {,total }}$, can be written as below.

$$
\eta_{\mathrm{m}, \text { total }}=\eta_{i}^{\mathrm{s}-\mathrm{cl}}=T|C|^{2}
$$

where $\eta_{1}^{s-c l}$ is the coupling efficiency between the source and the $\mathrm{HE}_{11}$ cladding mode found from Figure 2(a), $T$ is the lens transmittance which takes Fresnel reflection and beam truncation owing to the finite aperture of lensed fiber into account. $|C|^{2}$ indicates the limitation of coupling from a source with pure Gaussian mode to a hemispherical fiber with truncated and aberrated mode. Therefore, the coupling efficiencies can be calculated by using Eq. (1). Figure 3 shows the simulated and measured optimal coupling efficiencies versus beam waist. The calculated optimal coupling efficiencies is only $42 \%$ when the beam waist of the source is $1 \mu \mathrm{m}$. However, it markedly increases to 83 $\%$ for the beam waist of $3 \mu \mathrm{m}$ and becomes saturated to $85 \%$ for the beam waist larger than $4 \mu \mathrm{m}$. The low efficiency for the LD-to-fiber coupling results from the effects of aberration and beam truncation, which may be improved using a hyperbolic microlens design [5]. Note the measured optimal coupling efficiencies are $35 \%$ and 78 $\%$ for the beam waists of $1 \mu \mathrm{m}$ and $3 \mu \mathrm{m}$, respectively, which agree well with the simulated results based on the rayoptics.

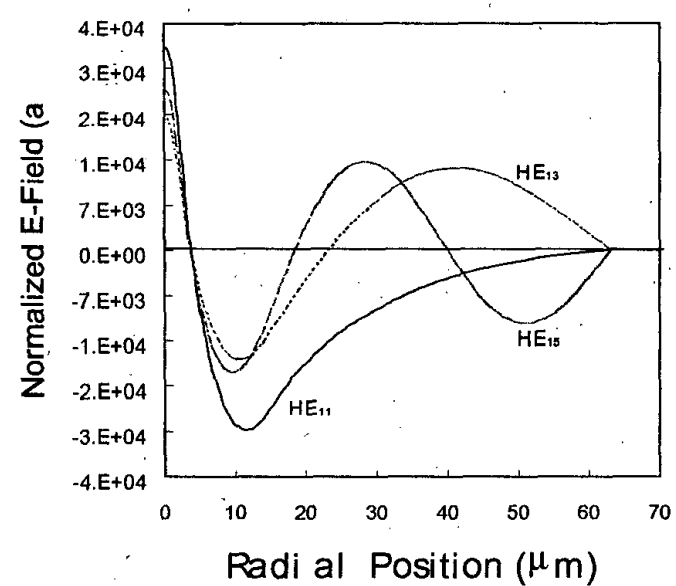

(a)

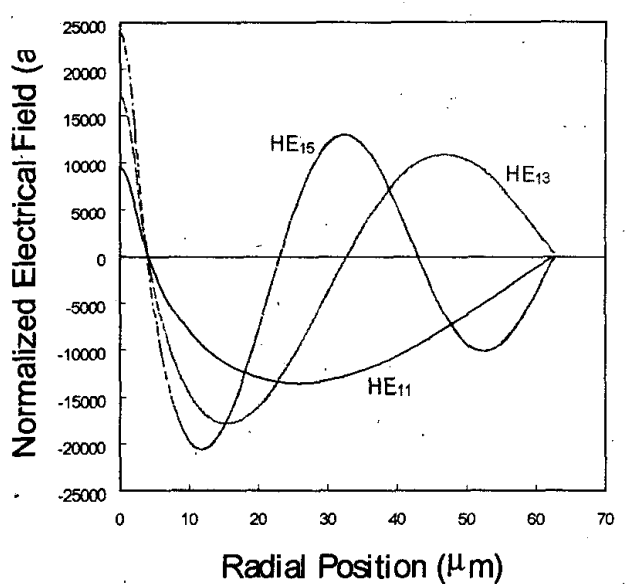

(b)

Fig. 2 Normalized electrical field distribution for a (a) DSF and (b) step-index SMF.

In summary, the fiber dependence and the efficiency limitation is analyzed for optical coupling using a lensed fiber integrated with a long-period fiber grating (LPFG). We found that a dispersion-shifted fiber is more efficient for the proposed coupling scheme than a standard single-mode fiber. The coupling efficiency seems to be strongly reduced for a small beam waist owing to the effects of fiber truncation and spherical aberration. A high coupling efficiency may be obtained for the LD-to-fiber coupling scheme if a hyperbolic lensed fiber is used. 


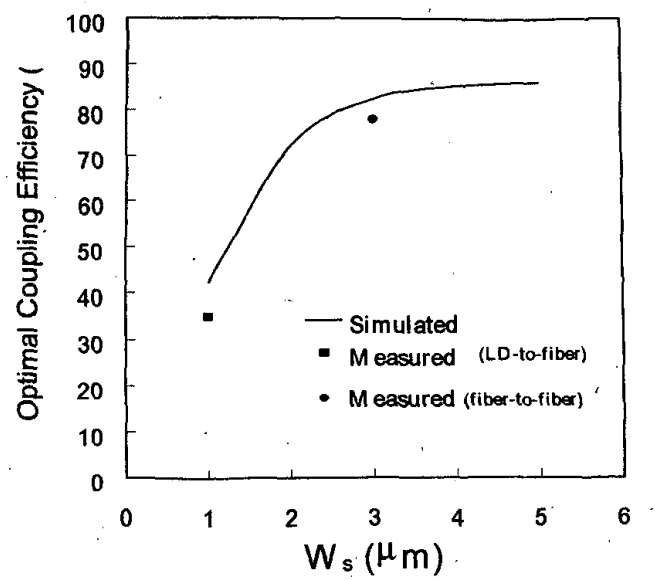

References

Fig. 3 Simulated and measured optimal coupling efficiency versus beam waist.

1. W.T. Chen and L.A. Wang, "Optical coupling between singlemode fibers by utilizing long-period fibre gratings," Electron. Lett., vol. 35 , pp. 421-423, 1999.

2. W. T. Chen and L. A. Wang, "Laser-to-fiber coupling scheme by utilizing a lensed fiber integrated with a long-period fiber grating," IEEE Photon. Technol. Lett., vol. 12, no. 5, pp. 501-503, 2000.

3. W. T. Chen and L. A. Wang, "Novel optical coupling utilizing a lensed fiber integrated with a long-period fiber grating," Appl. Opt. 39, $4490-4500,(2000)$.

4. T. Erdogan, "Cladding-mode resonances in short- and long- period fiber grating filters," J. Opt. Soc. Am. A 14, 1760-1773, (1997).

5. C. A. Edwards, H. M. Presby, and C. Dragone, "Ideal microlenses for laser to fiber coupling," J. Lightwave Technol. 11, 252-257, (1993). 